\title{
COVID-19 and diabetes: a review literature
}

\begin{abstract}
Background and aim: The new coronavirus, SARS-CoV-2 was identified as the pathogen responsible for causing the new coronavirus disease (COVID-19) and stated as a global pandemic by the World Health Organization. Most of the extreme and lethal cases with COVID-19 happened within the elderly or in patients with pre-existing comorbidities, such as diabetes mellitus (DM). In view of the above, the aim of this review was to investigate the scientific knowledge produced worldwide regarding the relationship between COVID-19 and diabetes mellitus.
\end{abstract}

Method: This article was conducted using the methodological framework of a scoping review, researched on PUBMED, CINAHL and SCOPUS database.

Results: A total of 12 studies were included. Six were developed in China, two in the United Kingdom, one in France, Spain, Kuwait and Ireland and all are in English.

Conclusion: Among the main conclusions that explain the relationship between the severity of the virus and the diabetic patient, the relationship of angiotensin-converting enzyme (ACE2) as a link between SARS-CoV-2 and target cells, hyperglycemia, HbA1c, whose level was associated in one of the studies with inflammatory markers, the synthesis and deregulated lipid clearance that compromises the immune system, the metabolic acidity that was given as a protective factor of the body against the infection of the new coronavirus and the "cytokine storm" are the ones that stand out. Future and clinical investigations to address gaps and unanswered questions about the relationship between COVID-19 and diabetes are of paramount importance.
Volume 5 Issue 4 - 2020

\author{
Anna Lucy Tavares de Oliveira,' Luiz Felipe \\ Lage Souza Camilo, ${ }^{2}$ Ravena Rieelly Araújo \\ Moura, ${ }^{2}$ Letícia Karoline Braga, ${ }^{2}$ Cassia \\ Regina Gontijo Gomes ${ }^{2}$ \\ 'Department of Nursing, Research and Institutode Santa Casa \\ de Belo Horizonte, Brazil \\ ${ }^{2}$ Department of Nursing, Faculty Pitágoras Betim, Brazil
}

Correspondence: Anna Lucy Tavares de Oliveira, Depatmnet of Nursing, Research and Institutode Santa Casa de Belo Horizonte, Brazil, Tel +5531994774819,

Email annalucy2@hotmail.com

Received: July 24, 2020 | Published: August 07, 2020

\section{Introduction}

Coronaviruses $(\mathrm{CoV})$ are structurally wrapped viruses with a single RNA genome, known to cause respiratory infections in humans and are the second leading cause of the common cold. In general, in most non-immunodeficient individuals, $\mathrm{CoV}$ infection leads to a mild upper respiratory infection. ${ }^{1}$

There are seven human coronaviruses (HCoVs) currently known, including the new coronavirus, SARS-CoV-2, which in December 2019 was identified as the pathogen responsible for causing the new coronavirus disease (COVID-19). On March 11, 2020, according to the World Health Organization, COVID-19 was stated as a global pandemic and on July 17, 2020, there were a total of 13,616,593 confirmed cases with 585,727 deaths worldwide. ${ }^{1,2}$

Most of these extreme and lethal cases with COVID-19 happened within the elderly or in patients with pre-existing comorbidities, such as diabetes mellitus (DM), ${ }^{3}$ thus suggesting that comorbidities are risk factors associated with disease severity and mortality. ${ }^{4}$

Patients with DM are more likely to acquire infections, especially influenza and pneumonia. Similarly, they are more susceptible to certain complications when infected with pathogens, as they undergo alterations in the pro-inflammatory state and constriction of the essential immune reactions.,

In DM this occurs, in part, because as the activated innate immune cells are aggregated in metabolic tissues, inflammatory mediators, as IL- $1 \beta$ and TNF $\alpha$, are discharged, promoting systemic insulin resistance and harm to $\beta$ cells. ${ }^{7}$

In view of the above, the aim of this review was to investigate the scientific knowledge produced worldwide regarding the relationship between COVID-19 and diabetes mellitus.

\section{Method}

To carry out this article, the methodology adopted was the Scoping Review, which maps the main concepts that underpin a research area and the main sources and types of evidence already available, determines the value of conducting a complete systematic review, summarizes and disseminates the results of the research and contributes to identify existing research gaps in the literature, such as that of the present study. ${ }^{8}$

The question that guided this study was: what is the scientific evidence produced about the increased risk of severity in patients with diabetes infected with COVID 19 ?

To select the articles, the online search was performed in the PUBMED, CINAHL and SCOPUS databases. The descriptors of the Medical Subject Headings (MeSH) used were: "COVID-19", "coronavirus", "diabetes mellitus" and "risk". The Boolean operators used were "AND" and "OR".

Inclusion criteria were defined as: research published in article form, in national and international journals, in Portuguese, English and Spanish; thatrelated diabetes and COVID 19; without temporal cutout; and that had titles and abstracts available and indexed in the databases. Exclusion criteria were: articles that did not address the aforementioned theme; review articles; book chapter; letters; editorials; and articles that the method was not clearly described.

\section{Results and discussion}

The sample consisted of 12 studies, ${ }^{1,9-19}$ in which all are in English and are from the year 2020. As for the country of publication, six were developed in China, two in the United Kingdom, one in France, Spain, Kuwait and Ireland. 
The analyzed studies showed that patients with diabetes who contract covid-19 are subject to a worse prognosis and increased mortality.

ACE2, the receptor for sars-CoV-2 to infect cells, is altered in patients with chronic diseases, such as diabetes, due to metabolic alterations, therefore increasing the chance of infection and severity of the disease in these patients, mentioned in the study by four authors ${ }^{1,10,15,18}$ as responsible for aggravating the evolution of covid-19 in these patients..$^{20,21}$

After being infected with the virus, the cells undergo necrosis or apoptosis, processes in which the activation of pro-inflammatory chemokines orcytokines, that are responsible for triggering inflammatory responses, and for the accumulation of defense cells at the site, occur. People with chronic hyperglycemia have a deregulated expression of ACE2, which leaves the cells more vulnerable to inflammatory effects, since in addition to the regulation of systemic arterial hypertension (SAH), this enzyme has protective effects, especially in relation to inflammation. ${ }^{1,22}$

The deregulated expression of ACE2 in pancreatic cells can cause a disturbance of their functions and lead to a deficiency in the production of hormones responsible for the regulatory process of glucose in the body, and may prevent the assembly of a coordinated and appropriate physiological response to the combat of the invading agent. ${ }^{10}$

Excess glucose in the bloodstream, which can be caused by the mechanisms mentioned above, may be the justification of the correlation between inflammatory markers and $\mathrm{HbAlc}$ or glycated hemoglobin in diabetic patients with Covid-19. Glycated hemoglobin is a result of the binding of the glucose molecule present in the bloodstream with circulating hemoglobin. Thus, high levels of HbAlc can refer us to a state of hyperglycemia, which according to the study by $\mathrm{Wu}$ et $\mathrm{al}^{23}$ is associated with a worse prognosis, given the relationship of the high glycemic level with the mechanism of infection of Covid-19. ${ }^{17}$

According to studies included in the sample, ${ }^{11,12,14,19}$ diabetic patients and/or patients with uncontrolled hyperglycemia had an increased mortality rate compared to other patients, as there is a proinflammatory state and a weaker immune response, since the function of defense cells is reduced in these patients, leading to a higher risk of secondary bacterial infections as well as virus-induced damage to multiple organs, such as liver and pancreatic injury. ${ }^{12}$

Hyperglycemia has multiple effects on the body, including immunosuppression, due to cellular and humoral immunity being impaired, as monocytes, macrophages, neutrophils, lymphocytes and $\mathrm{T}$ cells are decreased. It has also been proposed that the release of stress hormones, such as epinephrine and cortisol, lead to catecholaminemediated liver glycogenolysis, as well as direct sympathetic stimulation of glucose degradation, which would increase the level of hyperglycemia, and thus further aggravate its own effects, making it difficult to fight infections and diseases..$^{12,24,25}$

Obesity is a common risk factor for hyperglycemia, as it affects the kidneys, triggering intrarnal inflammation and recruiting immune cells through the so-called metainflamation, which is a low-grade inflammation mainly sustained by the excessive production of proinflammatory mediators by the adipose tissue. ${ }^{26}$

Two authors ${ }^{15,19}$ included in the sample explain that obesity may worsen the scenario in diabetic patients infected with covid-19, because according to Dommingo et al. ${ }^{19}$ obesity leads to a pulmonary surfactant dysfunction, while Al Sabah ${ }^{15}$ reports that it is linked to the synthesis and release of unregulated lipids, which may initiate or aggravate inflammations and lung lesions, worsening the evolution of COVID-19 in these patients.

The studies of Alkundi et al. ${ }^{11}$ showed that patients with diabetes, infected by COVID-19, suffering from Diabetic Ketoacidosis (DKA) are less likely to die compared to those who do not have the disease, and that children were generally asymptomatic or developed mild symptoms compared to other patients, since they have normal physiological metabolic acidosis, raising the hypothesis that metabolic acidity would actually be protective to the most severe types of COVID-19.

However, Palermo et al. $^{27}$ controversially states that in DKA, insulin insufficiency would trigger a cascade of non-adaptive physiological responses, including the formation of excessive ketone bodies that lead to metabolic acidosis and hyperglycemia, resulting in progressive hypovolemia and loss of electrolytes, constituting a deleterious and non-protective effect.

According to Finucane; Davenport ${ }^{10}$ COVID-19 and DM have implications on cytokines, stating that COVID-19 causes a cytokine storm, which is responsible for causing multiple organ failure and that DM modifies the cytokine profile and worsens an already unregulated immune reaction.

High glucose levels, captured by monocytes, induce viral replication and increase the amount of pro-inflammatory cytokines that act not only in the death of lung cells, but throughout the body through an uncontrolled immune response. ${ }^{28}$

The cytokine storm is followed by the immune system attacking the body due to an uncontrolled and widespread inflammatory response, which can cause multiple organ failure and in the most severe cases of SARS-CoV-2 infection, death. ${ }^{29}$

\section{Conclusion}

The pandemic has spread rapidly around the world, and has proven to be severe in some cases, such as diabetes, which should be assessed as a risk factor because it significantly increases morbidity and mortality. Future and clinical investigations to address gaps and unanswered questions about the relationship between COVID-19 and diabetes are of paramount importance to better understand the pathophysiological mechanisms involved in the association of these diseases that make severity more pronounced and to create specific therapeutic strategies to better serve them.

Among the main conclusions that explain the relationship between the severity of the virus and the diabetic patient, the relationship of angiotensin-converting enzyme (ACE2) as a link between SARSCoV-2 and target cells, hyperglycemia, HbAlc, whose level was associated in one of the studies with inflammatory markers, the synthesis and deregulated lipid clearance that compromises the immune system, the metabolic acidity that was given as a protective factor of the body against the infection of the new coronavirus and the "cytokine storm" are the ones that stand out.

\section{Acknowledgments}

None.

\section{Conflicts of interest}

The authors declare no conflicts of interest. 


\section{References}

1. Muniyappa R, Gubbi S. COVID-19 pandemic, coronaviruses, and diabetes mellitus. Am J Physiol Endocrinol Metab. 2020. 318:E736-E741.

2. World health organization. coronavirus disease 2019 (COVID-19) situation report-51.

3. Hussain A, Bhowmik B, do Vale Moreira NC. COVID-19 and diabetes: knowledge in progress. Diabetes Res Clin Pract. 2020. 162:108142.

4. Pal R, Bhansali A. COVID-19, diabetes mellitus and ACE2: The conundrum. Diabetes Res Clin Pract. 2020;162:108-132.

5. Knapp S. Diabetes and infection: is there a link?--A mini-review. Gerontology. 2013;2:99-104.

6. Gupta R, Hussain A, Misra A. Diabetes and COVID-19: evidence, current status and unanswered research questions. Eur J Clin Nutr. 2020;74:864870.

7. Yang J, Zheng Y, Gou X, et al. Prevalence of comorbidities and its effects in patients infected with SARS-CoV-2: a systematic review and metaanalysis. Int J Infect Dis. 2020;94:91-95.

8. Arksey H, O’Malley L. Scoping studies: towards a methodological framework. International Journal of Social Research Methodology. 2005;8(1):19-32.

9. Cariou B, Hadjadj S, Wargny M, et al. Phenotypic characteristics and prognosis of inpatients with COVID-19 and diabetes: the CORONADO study. Diabetologia. 2020;63:1500-1515.

10. Finucane FM, Davenport C. Coronavirus and obesity: could insulin resistance mediate the severity of Covid-19 infection?. Frontiers in public health. 2020;8:1-5.

11. Alkundi A, Mahmoud I, Musa A, et al. Clinical characteristics and outcomes of COVID-19 hospitalized patients with diabetes in the United Kingdom: A retrospective single centre study. Diabetes Research Clinical Practice. 2020;165:1-6.

12. Zhang Y, Li H, Zhang J, et al. The clinical characteristics and outcomes of patients with diabetes and secondary hyperglycaemia with coronavirus disease 2019: A single-centre, retrospective, observational study in Wuhan. Diabetes Obes Metab. 2020;8:1443-1454.

13. Zhang Y, Cui Y, Shen M, et al. Association of diabetes mellitus with disease severity and prognosis in COVID-19: A retrospective cohort study. Diabetes Res Clin Pract. 2020;16 (2020):1-8.

14. Yan Y, Yang Y, Wang F, et al. Clinical characteristics and outcomes of patients with severe covid-19 with diabetes. BMJ Open Diabetes Research and Care. 2020;; 8 (001343):1-9.

15. Al-Sabah S, Al-Haddad M, Al Youha S, et al. COVID-19: impact of obesity and diabetes in disease severity. medRxiv. 2020.
16. Guo W, Li M, Dong Y, et al. Diabetes is a risk factor for the progression and prognosis of COVID-19. Diabetes Metab Res Ver. 2020;(3319):1-9.

17. Wang Z, Du Z, Zhu F. Glycosylated hemoglobin is associated with systemic inflammation, hypercoagulability, and prognosis of COVID-19 patients. Diabetes Res Clin Pract. 2020;164:108214.

18. Bornstein SR, Rubino F, KhuntiK, et al. Practical recommendations for the management of diabetesin patients with COVID-19. The Lancet Diabetes and Endocrinology. 2020;8(6):546-550.

19. Domingo MP, Marazuela M, Giustina A. COVID-19 and endocrine diseases. A statement from the European Society of endocrinology. Endocrine. 2020;68:2-5.

20. de Abajo FJ, Rodríguez-Martín S, Lerma V, et al. Use of renin-angiotensinaldosterone system inhibitors and risk of COVID-19 requiring admission to hospital: a case-population study. The lancet. 2020;395(10238):17051714.

21. Zheng YY, Ma YT, Zhang JY, et al. COVID-19 and the cardiovascular system. Nat Rev Cardiol. 2020;17(5):259-260.

22. Cheng H, Wang Y, Wang GQ. Organ-protective effect of angiotensinconverting enzyme 2 and its effect on the prognosis of COVID-19. J Med Virol. 2020;92 (7):726-730.

23. Wu J, Huang J, Zhu G, et al. Elevation of blood glucose level predicts worse outcomes in hospitalized patients with COVID-19: a retrospective cohort study. BMJ Open Diabetes Res Care. 2020;8(1):e001476.

24. Kulcsar KA, Coleman CM, Beck SE, et al. Comorbid diabetes results in immune dysregulation and enhanced disease severity following MERSCoV infection. JCI Insight. 2019; 4(20):e131774.

25. Toro-Polo LM, Ortiz-Lozada RY, Chang-Grozo SL, et al. Glicemia na admissão e mortalidade em unidade de terapia intensiva pediátrica. Rev Bras Ter Intensiva. 2018; 30:471-478.

26. Brandão SCS, Silva ETAGBB, Melo LMMP, et al. Covid-19, imunidade, endotélio e coagulação:compreenda a interação. E-book. 2020;1:1-66.

27. Palermo NE, Sadhu AR, McDonnell ME. Diabetic ketoacidosis in COVID-19: unique concerns and considerations. J Clin Endocrinol Metab. 2020;105:1-11.

28. Codo AC, Davanzo GG, Monteiro LB, et al. Elevated glucose levels favor SARS-CoV-2 infection and monocyte response through a HIF-1 $\alpha$ / glycolysis dependent axis. Cell Metabolism. 2020.

29. Coperchini F, Chiovato L, Croce L, et al. The cytokine storm in COVID-19: An overview of the involvement of the chemokine/chemokine-receptor system. Cytokine Growth Factor Rev. 2020;53:25-32. 